\title{
Effect of low-load resistance training with different degrees of blood flow restriction in patients with knee osteoarthritis: study protocol for a randomized trial
}

\author{
Hao-Nan Wang ${ }^{\dagger}$, Yan Chen ${ }^{\dagger}$, Lin Cheng, Shen-Tao Wang, De-Xin Hu, Li-Na Wang and Guo-Xin Ni ${ }^{*}$ (D
}

\begin{abstract}
Background: Knee osteoarthritis (KOA) is a common degenerative disease that causes pain, functional impairment, and reduced quality of life. Resistance training is considered as an effective approach to reduce the risk of muscle weakness in patients with KOA. Blood flow restriction (BFR) with low-load resistance training has better clinical outcomes than low-load resistance training alone. However, the degree of BFR which works more effectively with low-load resistance training has not been determined. The purpose of this study is to evaluate the effectiveness of different degrees of BFR with low-load resistance training in patients with KOA on pain, self-reported function, physical function performance, muscle strength, muscle thickness, and quality of life.

Methods: This is a study protocol for a randomized, controlled trial with blinded participants. One hundred individuals will be indiscriminately assigned into the following groups: two training groups with a BFR at 40\% and $80 \%$ limb occlusion pressure (LOP), a training group without BFR, and a health education group. The three intervention groups will perform strength training for the quadriceps muscles twice a week for 12 weeks, while the health education group will attend sessions once a week for 12 weeks. The primary outcome is pain. The secondary outcomes include self-reported function, physical function performance, muscle strength of the knee extensors, muscle mass of the quadriceps, quality of life, and adverse events. Intention-to-treat analysis will be conducted for individuals who withdraw during the trial.
\end{abstract}

Discussion: Previous studies have shown that BFR with low-load resistance training is more effective than low-load resistance training alone; however, a high degree of BFR may cause discomfort during training. If a 40\% LOP for BFR could produce similar clinical outcomes as an $80 \%$ LOP for BFR, resistance training with a low degree of BFR can be chosen for patients with KOA who are unbearable for a high degree of BFR.

Trial registration: Chinese Clinical Trial Registry ChiCTR2000037859 (http://www.chictr.org.cn/edit.aspx?pid=59956 \&htm=4). Registered on 2 September 2020

Keywords: Knee osteoarthritis, Blood flow restriction, Vascular occlusion, Resistance training, Rehabilitation

\footnotetext{
* Correspondence: niquoxin@bsu.edu.cn

${ }^{\dagger}$ Hao-Nan Wang and Yan Chen contributed equally to this work.

School of Sport Medicine and Rehabilitation, Beijing Sport University, No. 48

Xinxi Road, Haidian District, Beijing 100084, China
}

C C The Author(s). 2021 Open Access This article is licensed under a Creative Commons Attribution 4.0 International License, which permits use, sharing, adaptation, distribution and reproduction in any medium or format, as long as you give appropriate credit to the original author(s) and the source, provide a link to the Creative Commons licence, and indicate if changes were made. The images or other third party material in this article are included in the article's Creative Commons licence, unless indicated otherwise in a credit line to the material. If material is not included in the article's Creative Commons licence and your intended use is not permitted by statutory regulation or exceeds the permitted use, you will need to obtain permission directly from the copyright holder. To view a copy of this licence, visit http://creativecommons.org/licenses/by/4.0/. The Creative Commons Public Domain Dedication waiver (http://creativecommons.org/publicdomain/zero/1.0/) applies to the data made available in this article, unless otherwise stated in a credit line to the data. 


\section{Background}

Knee osteoarthritis (KOA) is one of the most common chronic musculoskeletal diseases. It can develop the deterioration in the subchondral bone, cartilage, synovium, and menisci [1], resulting in pain, stiffness, impaired functioning in daily activities, and a decline in the quality of life (QoL). According to an epidemiological survey, it is estimated that the prevalence of symptomatic radiographic KOA among older people is $12.1 \%$ in the USA [2] and $8.1 \%$ in China [3], and these figures are manifesting an upward trajectory. Due to its high prevalence, KOA may cause tremendous economic burdens on health services, such as conventional interventions and joint replacements [4]. Therefore, primary and secondary prevention programs are necessary to minimize social and personal costs.

Multiple risk factors may contribute to the development of KOA, such as age, sex, obesity, and joint factors; unfortunately, the link between these factors and KOA remains unclear [5-7]. Nevertheless, muscle weakness, especially in the quadriceps, is considered both a vital and a modifiable risk factor for KOA [8]. Previous studies have shown that muscle weakness is strongly associated with the incidence [8] and progression [9] of KOA, as well as the physical function and knee pain of individuals [10]. Moreover, muscle mass in the lower limbs is independently influenced by the presence of KOA [11] and related to the severity of symptoms in patients [12].

The main approach to improve muscle strength and mass is resistance training (RT). RT can decrease the risk of KOA by increasing muscle strength and muscular hypertrophy [13]. Based on the recommendations of the American College of Sports Medicine, a resistance load of $60-80 \%$ of an individual's one-repetition maximum $(1 \mathrm{RM})$ is necessary to achieve muscle hypertrophy and improvements in muscular strength [14]. However, high-load resistance training (HLRT) can aggravate pain and joint deterioration in patients [15], resulting in decreased compliance to therapeutic exercises and a slower rehabilitation process.

Blood flow restriction (BFR) training has drawn the attention of clinicians and physiotherapists in the field of musculoskeletal rehabilitation, as it is believed to be an alternative approach to HLRT. In brief, a pneumatized cuff or tourniquet is used to block partial arterial blood flow to the limb, and this is combined with $20-30 \%$ 1RM low-load resistance training (LLRT). By creating a state of ischemia in the limbs, BFR training will produce a stronger physiological metabolic stress that includes growth hormones $[16,17]$ and increased recruitment of type II muscle fibers [18]. Thus, even though the load is relatively low during BFR training, it can still bring increases in muscle strength and mass, similar to the results obtained by HLRT. More importantly, BFR training requires a lower external load, which is beneficial in decreasing the joint loading and tolerance of RT.

Previous studies have found that BFR combined with LLRT yields better improvements in muscle strength compared with LLRT alone in patients with a risk of KOA $[19,20]$, while its effectiveness in increasing muscle strength, muscle mass, and function in patients with KOA is similar to that of HLRT [21, 22]. Nonetheless, it remains unclear whether the degree of BFR will influence the effectiveness of BFR with LLRT. Many factors contribute to the degree of BFR, including systolic blood pressure, diastolic blood pressure, sex, limb circumference, limb length, and cuff width $[23,24]$. The application of limb occlusion pressure (LOP) can produce a more precise degree of arterial occlusion for different individuals compared with absolute air pressure. Only one previous trial has used the percent of LOP to identify the pressure for BFR training in patients with KOA [22]. Notably, 70\% LOP BFR training has a similar effect in increasing muscle strength, quadriceps muscle mass, and function compared with HLRT. However, it has been shown that BFR pressures with $40 \%$ LOP are as effective as $80 \%$ LOP [15] in the acute response of muscles. Nonetheless, the ability to achieve a chronic response with BFR remains unclear, especially for patients with KOA. Additionally, while a higher LOP promotes more pain during exercise with BFR training [25], it seems that pressure with $40 \%$ LOP may be more comfortable for individuals compared with $80 \%$ LOP, making it beneficial in eliciting compliance to the intervention program.

It has shown in previous studies that the effectiveness of BFR with LLRT is not conclusive compared with LLRT alone [26]. For instance, Ferraz et al. demonstrated that LLRT with BFR is more effective in relieving pain compared with LLRT alone [22], while another study suggested that there was no difference between the two approaches [19]. Therefore, in this study, we set a group of LLRT without BFR to investigate whether LLRT with lower LOP is more effective than LLRT alone. Due to the fact that health education constitutes a minimized intervention that is nonetheless important for KOA [27], we set health education as a control group for comparison with the other three groups. The aim of this study is to investigate the efficacy of LLRT with different degrees of BFR in patients with KOA. Our measures will include pain, self-reported function, physical functional performance, muscular strength, muscle thickness, and QoL. We will also observe any adverse events to verify the safety of BFR training during the study.

Hypotheses:

1. LLRT, LLRT with low BFR (40\% LOP), and LLRT with high BFR (80\% LOP) will all be more effective than health education. 
2. Both LLRT with $40 \%$ BFR and $80 \%$ BFR will be more effective than LLRT alone, but there will be no difference between the two levels of BFR.

\section{Methods}

\section{Study design}

This study will be a prospective, single-blind, randomized controlled trial conducted in Beijing Chaoyang Hospital. The measurements of several outcomes will be made before and after the interventions and 24 weeks after the interventions begin (Fig. 1). This study was approved by the Ethics Committee of Beijing Sport University (Ethics Approval No. 2020108H) and registered at the Chinese Clinical Trial Registry (ChiCTR2000037859). We designed this study using the Standard Protocol Items: Recommendations for Interventional Trials (SPIRIT) statement [28] (Fig. 2). The results will be reported in accordance with the CONsolidated Standards of Reporting Trials (CONSORT) guidelines [29]. The participants will be informed that the trial will not collect any biological specimens for storage. This process will be carried out by researchers blinded to group allocation.

\section{Participants}

In this study, we will recruit 100 male and female patients with a diagnosis of unilateral or bilateral KOA. For participants with bilateral KOA, the affected side with worse Kellgren-Lawrence grade will be identified as the affected leg. The participants will then be randomly assigned to different interventions. The recruitment of participants will conduct through targeted strategies, including social media (WeChat), websites, newspapers, and other community approaches. If an interested patient meets the eligibility criteria, then the informed consent process will be performed by researchers who are blinded to group allocation. All potential participants will be informed that the study will last for 6 months, that they have the right to withdraw at any time, and

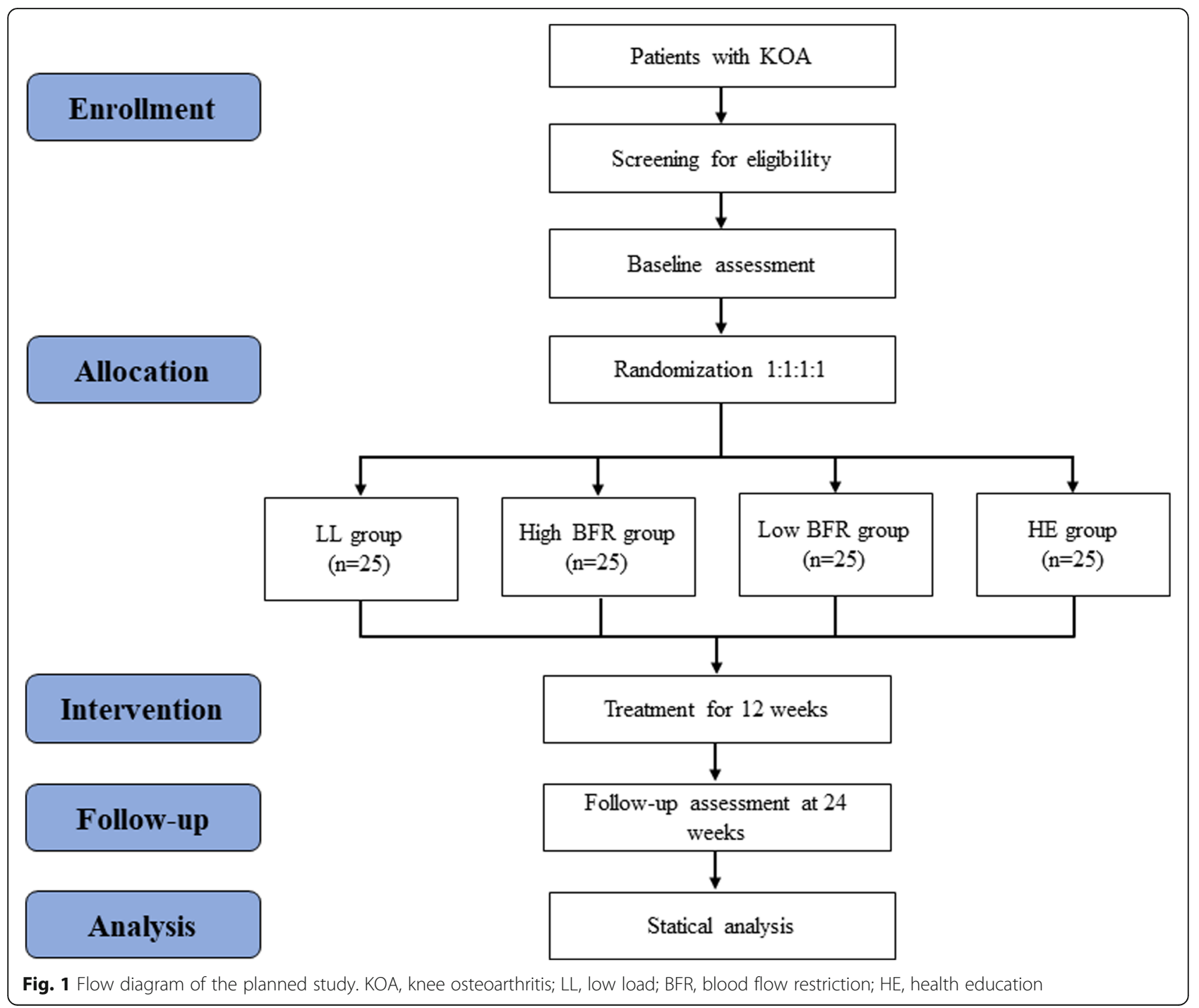




\begin{tabular}{|c|c|c|c|c|c|c|}
\hline & \multirow[b]{3}{*}{ ime point } & \multicolumn{5}{|c|}{ Study period } \\
\hline & & \multirow{2}{*}{$\begin{array}{l}\text { Screening } \\
\text { Week -2 }\end{array}$} & \multirow{2}{*}{$\begin{array}{l}\text { Baseline } \\
\text { Week } 0\end{array}$} & \multirow{2}{*}{$\begin{array}{c}\text { Twelve weeks } \\
\text { Week } 12\end{array}$} & \multicolumn{2}{|c|}{ Follow-up } \\
\hline & & & & & Week 13 & Week 24 \\
\hline \multirow{3}{*}{ Enrollment } & Eligibility screen & $x$ & & & & \\
\hline & Informed consent & $x$ & & & & \\
\hline & Allocation & & $x$ & & & \\
\hline \multirow{4}{*}{ Intervention } & LL group & & & $\longrightarrow$ & & \\
\hline & High BFR group & & & $\longleftrightarrow$ & & \\
\hline & Low BFR group & & & $\longleftrightarrow$ & & \\
\hline & Health education group & & & $\longleftrightarrow$ & & \\
\hline \multirow{7}{*}{ Assessment } & Pain & & $x$ & & $x$ & $x$ \\
\hline & Self-reported function & & $x$ & & $x$ & $x$ \\
\hline & Physical function performance & & $x$ & & $x$ & \\
\hline & Muscle strength & & $x$ & & $x$ & \\
\hline & Muscle thickness & & $x$ & & $x$ & \\
\hline & Quality of life & & $x$ & & $x$ & \\
\hline & Adverse events & & & & & \\
\hline
\end{tabular}

Fig. 2 Schedule of enrollment, intervention, and assessment (SPIRIT figure). LL, low load; BFR, blood flow restriction

that the trial will not collect any biological specimens for storage. If they are uncertain whether they will be able to participate for the entire 6-month period, we will provide regular healthcare for their KOA rather than include them in the formal study. All participants will be asked to provide written informed consent before the intervention. Figure 1 demonstrates the flow chart for the trial, which includes participant recruitment, eligibility screening, baseline measurements, randomization and allocation, intervention, and outcome assessments. The enrollment of participants, the randomization procedure, and the performance of various measurements will be conducted by four independent physiotherapists. A manual will be developed to standardize the administration of the subjective questionnaires and the objective performance tests.

\section{Inclusion criteria}

The inclusion criteria will be as follows: (1) 45-75 years of age, (2) unilateral or bilateral KOA diagnosed according to the American College of Rheumatology clinical classification system [14, 30], (3) radiologic confirmation of KOA demonstrating Kellgren-Lawrence grade II or III [31], (4) average pain intensity of 40 or more on a 100$\mathrm{mm}$ visual analogue scale (VAS) in the past week, and (5) adequate Mandarin language skills to complete the
Western Ontario and McMaster Universities Osteoarthritis Index (WOMAC) and the written informed consent.

\section{Exclusion criteria}

The exclusion criteria will be as follows: (1) a history of knee surgery or scheduled surgery, (2) a history of any invasive procedure in the affected knee, including arthroscopy or intra-articular injection in the past 12 months, (3) a history of physical therapy/physiotherapy or a strengthening procedure of the affected knee in the past 6 months, (4) use of nonsteroidal anti-inflammatory drugs in the past 3 months, (5) any neurological, heart, or vascular disease, such as blood coagulation disorders, (6) abnormal blood pressure (resting systolic blood pressure $[\mathrm{SBP}]>160$ or $<100 \mathrm{mmHg}$ or diastolic blood pressure $[\mathrm{DBP}]>100 \mathrm{mmHg}$ ), or (7) other acute or chronic disorders or psychiatric conditions that will affect physical or cognitive functions.

\section{Withdrawal criteria and management}

The KOA patients will be asked, or be allowed, to quit the study in the following cases: (1) the participants demand it and (2) there is a severe adverse event during the study. 


\section{Randomization and allocation concealment}

Eligible patients will be randomly assigned to one of the following groups at a ratio of 1:1:1:1: LL group, High BFR (80\% LOP) group, Low BFR (40\% LOP) group, and HE group. The research staff will independently use SAS software (SAS Institute, Cary, NC, USA) to generate a randomization sequence; this staff will not participate in the intervention or statistical analyses in the study. The number sequence will then be placed into a sequentially numbered, sealed, opaque envelope prior to the study by another independent research assistant who will also not participate in other parts of this study. Once the eligible patients with KOA complete the informed consent process, their demographic information and baseline measures will be recorded. Next, the research coordinator, who will not be involved in the measurement process, will have the authorization to open the envelopes in ascending order to determine a patient's intervention group assignment.

\section{Masking}

The participants will be informed that all of the interventions have proven efficiencies for their knee disorders, but it is still unknown which intervention works best. Moreover, they will be asked not to discuss their intervention content and group assignment at any time during the study. The intervention locations and times of intervention sessions will be separated for each individual. Unmasking will only be allowed in the case of severe adverse events and will be reported as part of the results of this study.

\section{Intervention}

All participants in the intervention groups will complete 24 exercise sessions over 12 weeks, with two sessions per week, as described in Table 1 . During the 12 weeks, the participants may continue their previous activities without aggravating their knee symptoms. In an individual exercise session, each group will perform a warm-up exercise to prevent injuries before the formal exercise protocol. Moreover, the pain intensity will be monitored using a VAS during the exercise, with some pain considered as acceptable. However, the load will be decreased by $20 \%$ if the pain intensity is higher than $20 \mathrm{~mm} / 100$ $\mathrm{mm}$ on the VAS [32]. During the period of intervention, all participants are not allowed to accept any other form of therapy, including medications.

Each group will conduct a warm-up by cycling for 10 minutes before the LLRT or BFR training. The leg press will be performed between 0 and $60^{\circ}$ of knee flexion, and the leg extension will be performed between 90 and $45^{\circ}$ of knee flexion [33]. In addition to thigh muscle strength training, distal joint, proximal joint, and core muscle strength training will also be performed. This is
Table 1 Treatment protocol performed by the LL group and the BFR groups

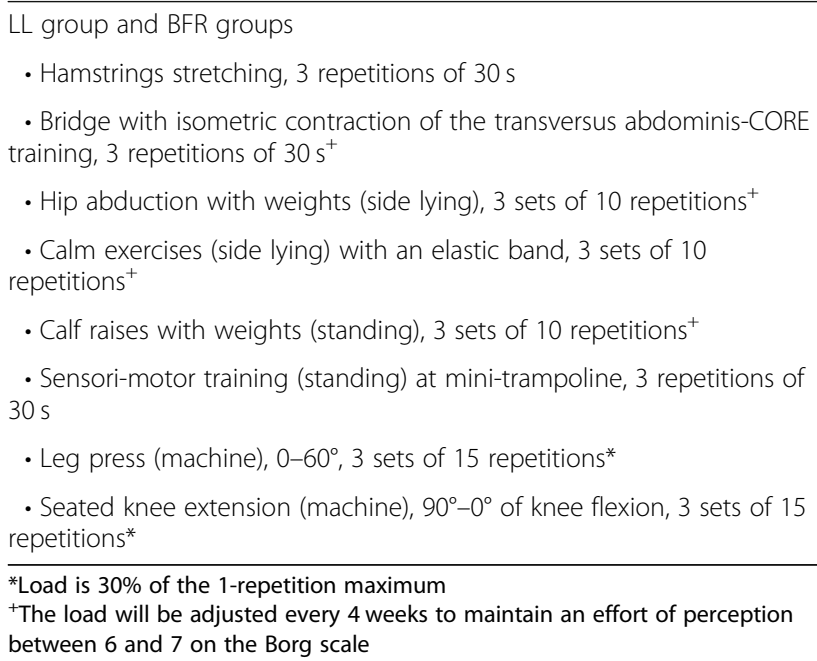

because current evidence has shown that biomechanical changes occur in patients with KOA [34], and a combined multi-joint strength exercise is potentially more effective than knee strength training alone in knee musculoskeletal disorders [35]. To avoid possible injuries during the training, we will provide a relatively integrated therapeutic exercise program. Thus, stretching exercises and core training will also be adopted in this study. During the treatment, we will adopt the 10-point Borg scale to monitor the perceived effort for several exercises [36], as shown in the detailed exercise program in Table 1.

The exercise load will be individually set for each participant and adjusted every 4 weeks by re-evaluating the participant's 1RM. Since a direct 1RM test could potentially cause knee pain or injury in patients with KOA, the $1 \mathrm{RM}$ will be estimated by performing a 7-10 RM test, which is the maximum load that an individual can complete for 7-10 repetitions. Previous studies have shown that the 7-10 RM test can accurately estimate the 1RM for leg press [37] and knee extension exercises [38]. The formula is as follows [39]: estimated 1RM = weight/(1.0278 - $0.0278 \times$ reps $)$.

\section{Blood flow restriction exercise groups}

All participants from the High BFR and Low BFR groups will be individually measured for the LOP and reevaluated every 2 weeks during the study. The LOP will be measured with the participants in relaxed and supine positions. The portable color Doppler ultrasound (LOGIQ e, General Electric Company, Boston, USA) will be positioned at the ankle to measure the pedal pulse. A pneumatic cuff (7-cm width and 56- $\mathrm{cm}$ length) will be placed on the proximal thigh of the participant and inflated until the pedal pulse vanishes on the Doppler 
ultrasound. We will then slowly deflate the cuff, and when the pedal pulse returns, we will record the LOP. The Low BFR group will perform at $40 \%$ LOP, while the High BFR group will perform at $80 \%$ LOP during the exercise. The loads for the leg press and knee extension exercises will be $30 \%$ of the 1RM (estimated by 7-10 RM). Furthermore, participants in the High BFR and Low BFR groups will perform one set of 30 repetitions (or until exhaustion) and three sets of 15 receptions with a 30-s interval between sets [20]. The LOP will be adjusted every time to maintain a similar degree of BFR for each individual.

\section{Low-load resistance exercise group}

The LL group will conduct a sham BFR, in which the pneumatic cuff will be placed on the proximal thigh of the participant with adequate space for two or more fingers between the thigh and cuff. However, no pressure will be applied to the pneumatic cuff. The exercise protocol of the LL group is the same as the BFR group. The LL group will perform three sets of 15 repetitions with 30\% 1RM and 30-s intervals between sets [19, 21, 32].

\section{Health education group}

The individuals in the health education group will attend sessions related to protecting their knee joints during daily life. The sessions will be conducted once a week for 12 weeks. Moreover, we will introduce the basic concept of KOA and the methods used to manage the risks of KOA through various articles from the Internet and leaflets.

\section{Outcome measures \\ Primary outcome measure}

\section{Pain}

A VAS will be used to evaluate pain intensity at rest and under maximum situations during the last week. The VAS comprises a line with a length of $100 \mathrm{~mm}$, in which " $0 \mathrm{~mm}$ " corresponds to no pain and " $100 \mathrm{~mm}$ " to the worst pain imaginable [40]. The VAS depicts pain effectively and is easily operated and widely applied in patients with KOA. The VAS will be evaluated at baseline and 12 and 24 weeks after the randomization of the participants. Notably, the minimal clinically important improvement for pain is about $20 \mathrm{~mm} / 100 \mathrm{~mm}$ [41].

\section{Secondary outcome measures}

1. Self-report function
The knee function self-report outcomes will be measured using the WOMAC. This is a 24-item self-report questionnaire that assesses joint pain, stiffness, and physical functions related to KOA [42]. The maximum WOMAC score is 120 , where a higher score indicates worse symptoms and functions of the knee. The Chinese version of the WOMAC has been shown to be both valid and reliable, as well as sensitive to changes in patients with KOA [43]. The WOMAC will be measured at baseline and 12 and 24 weeks after the randomization of the participants.

\section{Physical function performance}

The physical function performance will be assessed by the timed up and go (TUG) test [44]. Previous studies have found that the TUG test has a good intra- and inter-rater reliability ( 0.97 and 0.96 , respectively) for KOA patients with Kellgren-Lawrence grades 1-3 [45]. During the test, the subject is timed and required to independently rise from an armchair that is $45 \mathrm{~cm}$ in height, walk forward following a straight line for $3 \mathrm{~m}$, turn, walk back, and sit again. The average of three measurements will be used for analysis. The TUG will be measured at baseline and 12 weeks after the randomization of the participants.

\section{Muscle strength}

The quadriceps muscle strength will be evaluated by strength test of knee extension with an isokinetic test system (IsoMed 2000, D\&R Ferstl GmbH, Hemau, Germany). Before the strength test, the participants will be fastened onto a dynamometric chair in a $90^{\circ}$ sitting position, with the torso and thighs fixed by rigid belts. Then, the axis of the dynamometer will be adjusted in alignment with the center of the knee. Furthermore, the range of motion will be set individually for the participants by asking them to extend and flex their knees to maximum ranges. They will need to perform five constant flexion and extension motions using concentric contractions without a gravity-compensation model at three angular velocities of $60^{\circ} / \mathrm{s}, 90^{\circ} / \mathrm{s}$, and $120 \%$ [46]. During the test, the participants will be encouraged to perform at their maximum effort [47]. The data will be recorded and calculated as the peak torque in Newtonmeters, peak torque/body weight, and power in watts. Previous studies have demonstrated that the test-retest reliability for an isometric knee muscle strength assessment is 0.83 in patients with KOA [48]. The quadriceps muscle strength will be measured at baseline and 12 weeks after the randomization of the participants.

4. Muscle thickness 
The muscle thickness of the quadriceps will be measured using a portable color Doppler ultrasound [32, 49]. Intra-rater and inter-rater reliabilities have demonstrated good validation when evaluating the muscle by ultrasound. Furthermore, the correlations between the ultrasound and magnetic resonance imaging (MRI) scans for muscle thickness of the vastus medialis, vastus lateralis, and rectus femoris are $0.86,0.94$, and 0.86 , respectively $[50,51]$. During the test, the probe will be placed at the mid-belly of these three muscles without depressing the skin. Each muscle will be measured from the adipose tissue-muscle interface to the muscle-bone interface three times. The images will be saved and then averaged. The size of the quadriceps will be estimated as the sum of these three muscles. The muscle thickness will be measured at baseline and 12 weeks after the randomization of the participants.

\section{Quality of life}

The 36-item Short Form Health Survey (SF-36) is a brief self-report questionnaire with 36 questions relevant to QoL in eight health dimensions, including emotional aspects, physical aspects, social aspects, vitality, bodily pain, general health, physical functioning, and mental health in psychometric properties. It can be summarized in two health scores, namely, physical and mental components, where higher scores indicate a better health condition. Previous studies have indicated that the Chinese version of the SF-36 is a relative and valid questionnaire for the general population. The SF-36 will be measured at baseline and 12 weeks after the randomization of the participants.

\section{Adverse events}

All adverse events will be recorded throughout the entirety of the trial. Patients will be made aware of potential adverse events during the consent process and instructed to notify a researcher when adverse events occur. Accidental injuries will be collected through systematic participant spontaneous reporting. Potential adverse events of BFR or RT include muscle soreness, knee pain, subcutaneous hemorrhage, and numbness. Additionally, physiotherapists and related specialists will categorize adverse events as treatment-related or not and monitor the severity of the adverse events within 24 h. We will report all adverse events and describe whether they are related to the study.

\section{Sample size estimation}

Previous studies have shown that the effect size for pain is $0.38-0.49[52,53]$. The sample size was estimated by using G-Power software (version 3.1.9.6, Heinrich-
Heine-Universität Düsseldorf, Düsseldorf, Germany) with the following parameters: analysis of variance (ANOVA) with repeated measures, a type I error of $5 \%$ $(\alpha=0.05)$, a power of $95 \%(\beta=0.05)$, group numbers $=$ 4 , number of measurements $=3$, and effect size $=0.38$. The total sample size of this study should be a minimum of 84 participants. With a possible dropout rate of $15 \%$, it is estimated that a sample size of 25 patients per group will be needed to verify our study hypotheses for the primary outcomes.

\section{Statistical analyses}

All data will be expressed as mean \pm standard deviation (SD). A linear mixed model with repeated measures will be run to assess the data for fixed factors of the trial (LL group, High BFR group, Low BFR group, and HE group) and time (primary and secondary outcomes). Additionally, two-way ANOVAs will be used to analyze the differences between baseline, post-intervention, and followup measurements. Data from all subjects will be included in the statistical analyses, and the ITT approach will be applied to avoid disrupting the randomization of groups from dropping out through the trial. Any subject who started treatment will be included in the final outcome analysis regardless of whether he/she completed the study. Additionally, the missing data will be multiply imputed using chained equations with predictive mean matching, imputing data for each group separately. Estimates from 10 imputed data sets were combined using Rubin's rules [54]. The level of significance will be set at $P<0.05$ for all data. Statistical analyses will be performed using SPSS 22.0 (SPSS Inc., Chicago, USA).

\section{Data management, monitoring, and quality control}

The data will be carefully recorded by both printed and electronic case report forms (eCRFs). Only outcome assessors have access to the eCRFs, and all input data will be double-checked by two independent assessors. All data will be unmodifiable once input and checked through the eCRF. An independent Data and Safety Monitoring Board will be established from independent experts in orthopedics, physiotherapy, methodology, and statistics to review and interpret the trial data. The board will review the progress of the trial after 6 weeks and decide if premature closure of the study is required based solely on adverse events. The Institute of Sports and Health of China will be responsible for verifying the accuracy of the data. Only the statisticians will have access to the database to conduct final statistical analyses. The data collected from this trial will not be used in secondary or ancillary studies. In this trial, both online and on-site monitoring will be adopted to review the trial processes. The ethics committee of Beijing Sport University will monitor protocol violations weekly. The 
participants, ethics committee, and Chinese Clinical Trial Registry will be informed of any protocol modifications by email.

\section{Discussion}

BFR training has become a novel intervention for lower limb degeneration or post-surgical musculoskeletal disorders. However, there have been no consistent recommendations for BFR training in treating $\mathrm{KOA}$ in any guidelines until now. In this trial, we will investigate the efficacy of LLRT combined with different BFR pressures ( $0 \%, 40 \%$, and $80 \%$ LOP) on pain, self-reported function, physical functional performance, muscle strength, muscle mass, and QoL.

Although several studies have indicated that BFR combined with LLRT is more effective than LLRT alone [19, 20], the most efficacious degree of BFR has not been determined. If a 40\% LOP during BFR will produce similar clinical outcomes as an $80 \%$ LOP during BFR in patients with KOA, resistance using a lower LOP can be a good choice for those who are unbearable for high degree of LOP. Additionally, there has been no study comparing the effects of BFR training with a control group without an exercise intervention, and a complete placebo control is not applicable for patients with KOA. Thus, we will set up a control group (the health education group), which will make the study more rigorous and objective. We believe our study will provide evidence for the effectiveness of RT with BFR, which is important for clinicians in treating patients with KOA.

This trial meets the requirements of methodology for the application of randomization, allocation concealment, and ITT approach, as well as masking for patients. In this trial, there will be two sessions per week for 12 weeks, yielding a total of 24 exercise sessions for the exercise intervention groups. We will investigate the shortterm effects (12 weeks) of these four groups for all clinical outcomes and safety, as well as the mid-term (24 weeks) effects of pain and self-reported function.

We will use the percent of LOP to apply as similarly as possible the degree of BFR for individuals in each group. Moreover, the LOP will be tested before every session to avoid possible changes in the hemodynamics of the participants. Considering the potential placebo effect for patients in the LL resistance group, the BFR cuff will also be applied to the participants during the training, but the cuff will not be inflated. It is worth mentioning that only one study has monitored pain intensity during training and compared the differences between BFR training and HLRT [21]. In this study, we will evaluate the pain intensity during training compared to the tolerance to different degrees of BFR in patients with KOA.
Despite its strengths, this study has some potential limitations. First, due to the nature of the intervention, the physiotherapist cannot be fully masked during the research. Second, like other studies using BFR training, the LOP will be assessed at rest, and it may be altered by changes in body position and muscle contraction. Third, the intervention program consists of core training and stretching, in addition to quadriceps training, to provide better outcomes for patients with KOA. However, these interventions, especially the stretching exercises, may influence certain outcomes, such as muscle strength. At the end of this research, our results may provide more reliable evidence on the effectiveness of BFR training and identify the most appropriate degree of BFR in treating patients with KOA.

\section{Trial status}

The currently approved version of the protocol is version 1.0 dated March 2021. Recruitment is still in progress and will be completed by February 2022 .

\section{Abbreviations \\ KOA: Knee osteoarthritis; BFR: Blood flow restriction; LLRT: Low-load resistance training; HLRT: High-load resistance training; 1RM: One-repetition maximum; LOP: Limb occlusion pressure; SD: Standard deviation; SF-36: Short Form 36; VAS: Visual analog scale; TUG: Timed up and go; WOMAC: Western Ontario and McMaster Universities Osteoarthritis Index}

\section{Acknowledgements}

We would like to thank Xiaoxiong Zhao from the Beijing Chaoyang Hospital for their help in suggesting the study design and recruitment of participants.

Authors' contributions

HNW, LC, and GXN conceived and designed the trial. HNW, YC, and STW participated in the trial register, communication, and monitoring. DXH and

LNW participated in the design of statistical analysis. All authors contributed to drafting the manuscript and read and approved the final version.

\section{Funding}

This work was supported by the National Natural Science Foundation of China (81871848).

Availability of data and materials

The data and the relevant results in this study will be shared through academic conferences and scientific papers. The datasets can be obtained from the corresponding author upon reasonable request.

\section{Declarations}

Ethics approval and consent to participate

The study was approved by the ethics committee of the Beijing Sport University, China. Participants will sign a written informed consent before the intervention.

Consent for publication

Not applicable.

Competing interests

All authors declare that they have no competing interests. 
Received: 26 April 2021 Accepted: 17 December 2021

\section{Published online: 03 January 2022}

\section{References}

1. Sharma L. Osteoarthritis of the Knee. N Engl J Med. 2021;384(1):51-9. https://doi.org/10.1056/NEJMcp1903768.

2. Dillon CF, Rasch EK, Gu Q, Hirsch R. Prevalence of knee osteoarthritis in the United States: arthritis data from the Third National Health and Nutrition Examination Survey 1991-94. J Rheumatol. 2006:33(11):2271-9.

3. Tang X, Wang S, Zhan S, Niu J, Tao K, Zhang Y, et al. The prevalence of symptomatic knee osteoarthritis in China: results from the China Health and Retirement Longitudinal Study. Arthritis \& rheumatology (Hoboken, NJ). 2016;68:648-53.

4. Cross M, Smith E, Hoy D, Nolte S, Ackerman I, Fransen M, et al. The global burden of hip and knee osteoarthritis: estimates from the global burden of disease 2010 study. Ann Rheum Dis. 2014;73(7):1323-30. https://doi.org/1 0.1136/annrheumdis-2013-204763.

5. Neogi T, Zhang Y. Epidemiology of osteoarthritis. Rheum Dis Clin North Am. 2013;39(1):1-19. https://doi.org/10.1016/j.rdc.2012.10.004.

6. Vina ER, Kwoh CK. Epidemiology of osteoarthritis: literature update. Curr Opin Rheumatol. 2018;30(2):160-7. https://doi.org/10.1097/BOR. 0000000000000479.

7. Johnson VL, Hunter DJ. The epidemiology of osteoarthritis. Best Pract Res Clin Rheumatol. 2014;28(1):5-15. https://doi.org/10.1016/j.berh.2014.01.004

8. Takagi S, Omori G, Koga H, Endo K, Koga Y, Nawata A, et al. Quadriceps muscle weakness is related to increased risk of radiographic knee OA but not its progression in both women and men: the Matsudai Knee Osteoarthritis Survey. Knee Surg Sports Traumatol Arthrosc. 2018;26(9):260714. https://doi.org/10.1007/s00167-017-4551-5.

9. Dell'isola A, Wirth W, Steultjens M, Eckstein F, Culvenor AG. Knee extensor muscle weakness and radiographic knee osteoarthritis progression. Acta Orthop. 2018;89(4):406-11. https://doi.org/10.1080/17453674.2018.1464314.

10. Muraki S, Akune T, Teraguchi M, Kagotani R, Asai Y, Yoshida M, et al. Quadriceps muscle strength, radiographic knee osteoarthritis and knee pain: the ROAD study. BMC Musculoskelet Disord. 2015;16(1):305. https://doi.org/1 0.1186/s12891-015-0737-5.

11. Lee SY, Ro HJ, Chung SG, Kang SH, Seo KM, Kim DK. Low skeletal muscle mass in the lower limbs is independently associated to knee osteoarthritis. PLoS One. 2016;11(11):e0166385. https://doi.org/10.1371/journal.pone.01663 85.

12. Cheon $\mathrm{YH}$, Kim HO, Suh YS, Kim MG, Yoo WH, Kim RB, et al. Relationship between decreased lower extremity muscle mass and knee pain severity in both the general population and patients with knee osteoarthritis: Findings from the KNHANES V 1-2. PLoS One. 2017;12(3):e0173036. https://doi.org/1 0.1371/journal.pone.0173036.

13. Liao CD, Chen HC, Kuo YC, Tsauo JY, Huang SW, Liou TH. Effects of muscle strength training on muscle mass gain and hypertrophy in older adults with osteoarthritis: a systematic review and meta-analysis. Arthritis Care Res. 2020;72(12):1703-18. https://doi.org/10.1002/acr.24097.

14. American College of Sports Medicine position stand. Progression models in resistance training for healthy adults. Med Sci Sports Exerc. 2009;41(3):687708. https://doi.org/10.1249/MSS.0b013e3181915670.

15. Jan MH, Lin JJ, Liau JJ, Lin YF, Lin DH. Investigation of clinical effects of high- and low-resistance training for patients with knee osteoarthritis: a randomized controlled trial. Phys Ther. 2008;88(4):427-36. https://doi.org/1 $0.2522 /$ ptj.20060300.

16. Takarada Y, Nakamura Y, Aruga S, Onda T, Miyazaki S, Ishii N. Rapid increase in plasma growth hormone after low-intensity resistance exercise with vascular occlusion. J Appl Physiol (1985). 2000;88:61-5.

17. Reeves GV, Kraemer RR, Hollander DB, Clavier J, Thomas C, Francois M, et al. Comparison of hormone responses following light resistance exercise with partial vascular occlusion and moderately difficult resistance exercise without occlusion. J Appl Physiol (1985). 2006;101:1616-22.

18. Moore DR, Burgomaster KA, Schofield LM, Gibala MJ, Sale DG, Phillips SM. Neuromuscular adaptations in human muscle following low intensity resistance training with vascular occlusion. Eur J Appl Physiol. 2004;92(4-5): 399-406. https://doi.org/10.1007/s00421-004-1072-y.

19. Segal N, Davis MD, Mikesky AE. Efficacy of blood flow-restricted low-load resistance training for quadriceps strengthening in men at risk of symptomatic knee osteoarthritis. Geriatr Orthop Surg Rehabil. 2015;6(3):1607. https://doi.org/10.1177/2151458515583088.
20. Segal NA, Williams GN, Davis MC, Wallace RB, Mikesky AE. Efficacy of blood flow-restricted, low-load resistance training in women with risk factors for symptomatic knee osteoarthritis. PM R. 2015;7(4):376-84. https://doi.org/10.1 016/j.pmrj.2014.09.014

21. Bryk FF, Dos Reis AC, Fingerhut D, Araujo T, Schutzer M, Cury Rde P, et al. Exercises with partial vascular occlusion in patients with knee osteoarthritis: a randomized clinical trial. Knee Surg Sports Traumatol Arthrosc. 2016;24(5): 1580-6. https://doi.org/10.1007/s00167-016-4064-7.

22. Ferraz RB, Gualano B, Rodrigues R, Kurimori CO, Fuller R, Lima FR, et al. Benefits of resistance training with blood flow restriction in knee osteoarthritis. Med Sci Sports Exerc. 2018;50(5):897-905. https://doi.org/1 0.1249/MSS.0000000000001530

23. Jessee MB, Buckner SL, Dankel SJ, Counts BR, Abe T, Loenneke JP. The influence of cuff width, sex, and race on arterial occlusion: implications for blood flow restriction research. Sports Med. 2016;46(6):913-21. https://doi. org/10.1007/s40279-016-0473-5.

24. Loenneke JP, Fahs CA, Rossow LM, Sherk VD, Thiebaud RS, Abe T, et al Effects of cuff width on arterial occlusion: implications for blood flow restricted exercise. Eur J Appl Physiol. 2012;112(8):2903-12. https://doi.org/1 0.1007/s00421-011-2266-8.

25. Soligon SD, Lixandrão ME, Biazon T, Angleri V, Roschel H, Libardi CA. Lower occlusion pressure during resistance exercise with blood-flow restriction promotes lower pain and perception of exercise compared to higher occlusion pressure when the total training volume is equalized. Physiol Int. 2018;105(3):276-84. https://doi.org/10.1556/2060.105.2018.3.18.

26. Wang HN, Chen Y, Cheng L, Cai YH, Li W, Ni GX. Efficacy and safety of blood flow restriction training in patients with knee osteoarthritis: a systematic review and meta-analysis. Arthritis Care Res. 2021. https://doi. org/10.1002/acr.24787

27. Gay C, Chabaud A, Guilley E, Coudeyre E. Educating patients about the benefits of physical activity and exercise for their hip and knee osteoarthritis. Systematic literature review. Ann Phys Rehabil Med. 2016; 59(3):174-83. https://doi.org/10.1016/j.rehab.2016.02.005.

28. Chan AW, Tetzlaff JM, Altman DG, Laupacis A, Gøtzsche PC, Krleža-Jerić K, et al. SPIRIT 2013 statement: defining standard protocol items for clinical trials. Ann Intern Med. 2013;158(3):200-7. https://doi.org/10.7326/0003-481 9-158-3-201302050-00583.

29. Zwarenstein M, Treweek S, Gagnier JJ, Altman DG, Tunis S, Haynes B, et al. Improving the reporting of pragmatic trials: an extension of the CONSORT statement. BMJ (Clinical research ed). 2008;337:a2390.

30. Fernandes L, Hagen KB, Bijlsma JW, Andreassen O, Christensen P, Conaghan $P G$, et al. EULAR recommendations for the non-pharmacological core management of hip and knee osteoarthritis. Ann Rheum Dis. 2013;72(7): 1125-35. https://doi.org/10.1136/annrheumdis-2012-202745.

31. Kellgren JH, Lawrence JS. Radiological assessment of osteo-arthrosis. Ann Rheum Dis. 1957;16(4):494-502. https://doi.org/10.1136/ard.16.4.494.

32. Giles L, Webster KE, McClelland J, Cook JL. Quadriceps strengthening with and without blood flow restriction in the treatment of patellofemoral pain: a double-blind randomised trial. Br J Sports Med. 2017;51(23):1688-94. https://doi.org/10.1136/bjsports-2016-096329.

33. Powers CM, Ho KY, Chen YJ, Souza RB, Farrokhi S. Patellofemoral joint stress during weight-bearing and non-weight-bearing quadriceps exercises. J Orthop Sports Phys Ther. 2014;44(5):320-7. https://doi.org/10.2519/jospt.2 014.4936 .

34. Astephen JL, Deluzio KJ, Caldwell GE, Dunbar MJ. Biomechanical changes at the hip, knee, and ankle joints during gait are associated with knee osteoarthritis severity. J Orthop Res. 2008;26(3):332-41. https://doi.org/10.1 002/jor.20496

35. Hislop AC, Collins NJ, Tucker K, Deasy M, Semciw Al. Does adding hip exercises to quadriceps exercises result in superior outcomes in pain, function and quality of life for people with knee osteoarthritis? A systematic review and meta-analysis. Br J Sports Med. 2020;54(5):263-71. https://doi. org/10.1136/bjsports-2018-099683.

36. Thomas SG, Pagura SM, Kennedy D. Physical activity and its relationship to physical performance in patients with end stage knee osteoarthritis. J Orthop Sports Phys Ther. 2003;33(12):745-54. https://doi.org/10.2519/jospt.2 003.33.12.745

37. Reynolds JM, Gordon TJ, Robergs RA. Prediction of one repetition maximum strength from multiple repetition maximum testing and anthropometry. J Strength Cond Res. 2006;20(3):584-92. 
38. Abadie BR, Wentworth MC. Prediction of one repetition maximal strength from a 5-10 repetition submaximal strength test in college-aged females. J. Exerc. Physiol. Online. 2000;3(3):1-8.

39. McNair PJ, Colvin M, Reid D. Predicting maximal strength of quadriceps from submaximal performance in individuals with knee joint osteoarthritis. Arthritis Care Res. 2011;63(2):216-22. https://doi.org/10.1002/acr.20368.

40. Wewers ME, Lowe NK. A critical review of visual analogue scales in the measurement of clinical phenomena. Res Nurs Health. 1990;13(4):227-36. https://doi.org/10.1002/nur.4770130405.

41. Tubach F, Ravaud P, Baron G, Falissard B, Logeart I, Bellamy N, et al. Evaluation of clinically relevant changes in patient reported outcomes in knee and hip osteoarthritis: the minimal clinically important improvement. Ann Rheum Dis. 2005;64(1):29-33. https://doi.org/10.1136/ard.2004.022905.

42. Bellamy N, Buchanan WW, Goldsmith CH, Campbell J, Stitt LW. Validation study of WOMAC: a health status instrument for measuring clinically important patient relevant outcomes to antirheumatic drug therapy in patients with osteoarthritis of the hip or knee. J Rheumatol. 1988;15(12): 1833-40.

43. Symonds T, Hughes B, Liao S, Ang Q, Bellamy N. Validation of the Chinese Western Ontario and McMaster Universities Osteoarthritis Index in patients from Mainland China with osteoarthritis of the knee. Arthritis Care Res. 2015; 67(11):1553-60. https://doi.org/10.1002/acr.22631.

44. Podsiadlo D, Richardson S. The timed "Up \& Go": a test of basic functional mobility for frail elderly persons. J Am Geriatr Soc. 1991;39(2):142-8. https:// doi.org/10.1111/j.1532-5415.1991.tb01616.x.

45. Alghadir A, Anwer S, Brismée JM. The reliability and minimal detectable change of Timed Up and Go test in individuals with grade 1-3 knee osteoarthritis. BMC Musculoskelet Disord. 2015;16(1):174. https://doi.org/1 0.1186/s12891-015-0637-8.

46. Harper SA, Roberts LM, Layne AS, Jaeger BC, Gardner AK, Sibille KT, et al. Blood-flow restriction resistance exercise for older adults with knee osteoarthritis: a pilot randomized clinical trial. J Clin Med. 2019;8(2). https:// doi.org/10.3390/jcm8020265

47. Hall M, Hinman RS, Wrigley TV, Kasza J, Lim BW, Bennell KL. Knee extensor strength gains mediate symptom improvement in knee osteoarthritis: secondary analysis of a randomised controlled trial. Osteoarthr Cartil. 2018; 26(4):495-500. https://doi.org/10.1016/j.joca.2018.01.018.

48. Wessel J. Isometric strength measurements of knee extensors in women with osteoarthritis of the knee. J Rheumatol. 1996;23(2):328-31.

49. Kim D, Loenneke JP, Thiebaud RS, Abe T, Bemben MG. The acute muscular effects of cycling with and without different degrees of blood flow restriction. Acta Physiol Hung. 2015;102(4):428-41. https://doi.org/10.1556/03 6.102.2015.4.10.

50. Giles LS, Webster KE, McClelland JA, Cook J. Can ultrasound measurements of muscle thickness be used to measure the size of individual quadriceps muscles in people with patellofemoral pain? Phys Ther Sport. 2015;16(1):4552. https://doi.org/10.1016/j.ptsp.2014.04.002

51. Giles LS, Webster KE, McClelland JA, Cook J. Atrophy of the quadriceps is not isolated to the vastus medialis oblique in individuals with patellofemoral pain. J Orthop Sports Phys Ther. 2015;45(8):613-9. https://doi. org/10.2519/jospt.2015.5852.

52. Fransen M, McConnell S, Harmer AR, Van der Esch M, Simic M, Bennell KL. Exercise for osteoarthritis of the knee: a Cochrane systematic review. $\mathrm{Br} J$ Sports Med. 2015;49(24):1554-7. https://doi.org/10.1136/bjsports-2015095424

53. Jansen MJ, Viechtbauer W, Lenssen AF, Hendriks EJ, de Bie RA. Strength training alone, exercise therapy alone, and exercise therapy with passive manual mobilisation each reduce pain and disability in people with knee osteoarthritis: a systematic review. J Physiother. 2011;57(1):11-20. https://doi. org/10.1016/S1836-9553(11)70002-9.

54. White IR, Royston P, Wood AM. Multiple imputation using chained equations: Issues and guidance for practice. Stat Med. 2011;30(4):377-99. https://doi.org/10.1002/sim.4067.

\section{Publisher's Note}

Springer Nature remains neutral with regard to jurisdictional claims in published maps and institutional affiliations.

Ready to submit your research? Choose BMC and benefit from:

- fast, convenient online submission

- thorough peer review by experienced researchers in your field

- rapid publication on acceptance

- support for research data, including large and complex data types

- gold Open Access which fosters wider collaboration and increased citations

- maximum visibility for your research: over $100 \mathrm{M}$ website views per year

At BMC, research is always in progress.

Learn more biomedcentral.com/submissions 